\title{
The Variations of the $\mathrm{H} \alpha$ line of HD199178
}

\author{
Lauri Jetsu ${ }^{1}$, Igor Savanov ${ }^{2}$, Ilkka Tuominen ${ }^{1}$ \\ ${ }^{1}$ Observatory and Astrophysics Laboratory, University of Helsinki, \\ Tähtitorninmäki, SF-00130 Helsinki, Finland \\ ${ }^{2}$ Crimean Astrophysical Observatory, P/O Nauchny, 334413 Crimea, \\ USSR
}

\begin{abstract}
Changes of emission cause variations of the $\mathrm{H} \alpha$ absorption line of $\mathrm{HD} 199178$. The correlation with the simultaneous light curve may depend on the relative strength of the axisymmetric and non-axisymmetric components of the evolving spot configurations.
\end{abstract}

\section{Introduction}

Bopp and Stencel (1981) defined a new class of objects: FK Comae-type stars, which are apparently single, rapidly rotating, late-type giants of strong chromospheric activity. HD 199178 (G5 III-IV; $v \sin i=80 \pm 3.3 \mathrm{~km} \mathrm{~s}^{-1} ; P_{\text {phot }}=$ $3.337484 \pm 0.000043$ ) is one of the original candidates of this class (Herbig, 1958; Huenemoerder, 1986; Jetsu et al., 1990a,b). A double period spot cycle of was proposed for HD 199178 by Jetsu et al. (1990a). The FK Comae stars show shortterm changes of the $\mathrm{H} \alpha$ line profile (e.g. Walter and Basri, 1982; Bopp et al., 1984; Huenemoerder, 1986). This paper presents some results of a search for correlations between the changes of $\mathrm{H} \alpha$ line profile and the simultaneous rotational modulation of brightness, i.e. spot activity. Spectroscopic observations were made at the Crimean Astrophysical Observatory during two seasons: September, 1986 and October - November, 1989. The nearly simultaneous photometry is from Jetsu et al. $(1990 \mathrm{a}, \mathrm{b}, \mathrm{c})$. 


\section{The $\mathrm{H} \alpha$ absorption and emission}

The parameters derived from the $\mathrm{H} \alpha$ absorption line profile were: the equivalent width of absorption $E W$, the ratio of blue absorption equivalent width to red absorption equivalent width $E W_{B} / E W_{R}$ and the centre $C$ and peak $P$ of a gaussian fit to the absorption line profile, which are plotted in Fig. 1. as a function of phase derived from the ephemeris of Jetsu et al. (1990b).
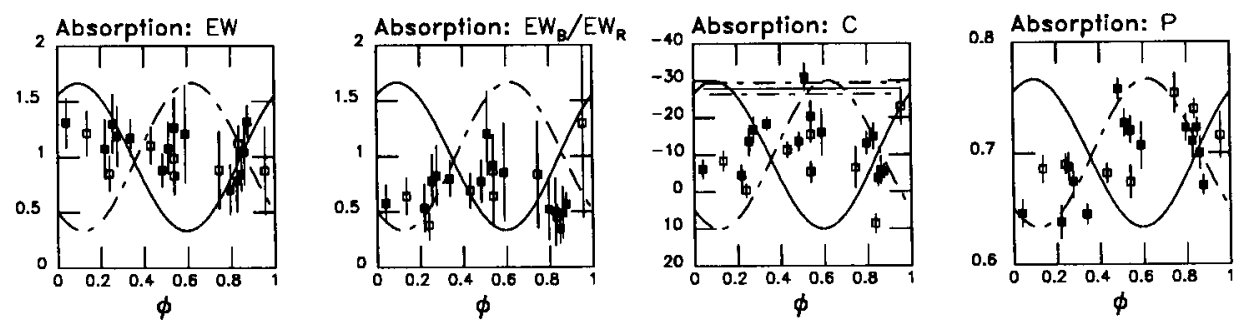

Fig. 1. The parameters $E W, E W_{B} / E W_{R}, C$ and $P$ : the results during 1986 are denoted by filled squares and those during 1989 by open squares. The light curves in V during both seasons are outlined in every picture on an arbitrary scale (1986: dashed curve, 1989: continuous curve). The radial velocity of HD 199178 published by Huenemoerder (1986) is shown as a straight line with error limits indicated in the second picture from the right.

An estimate of the chromospheric emission was obtained by substracting the artificially broadened spectrum of the chromospherically inactive giant $\rho$ Cyg (G8 III) from the spectra of HD 199178 (G5 III-IV). The parameters of emission (Fig. 2.) derived during both season were: the equivalent width of emission $E W_{e m}$, the ratio of blue emission equivalent width to the red $E W_{B, e m} / E W_{R, e m}$, the peak intensity of emission $P_{e m}$ and the respective of wavelength of this peak $C_{e m}$. (Note that the amplitudes of the modulation of both $C$ and $C_{e m}$ during 1986 are of the same order as those published by Neff et al. (1988) for the variation of the centre of $\mathrm{Mg}$ II $k$ line).

\section{Conclusions}

During 1986 the parameters $E W_{B} / E W_{R}, E W_{B, e m} / E W_{R, e m}, C, C_{e m}, P$ and $P_{e m}$ do show more than marginal rotational modulation, and consequently quite a good correlation with the simultaneous light curve. During 1989 no clearly rotational modulation of emission or absorption is present, which naturally leads to a nonexistent correlation with the light curve.

However, the mean brightness during 1989 is considerably lower $\left(0.06^{m}\right)$ than during 1986. The difference is about $60 \%$ of the peak to peak amplitude of varia- 

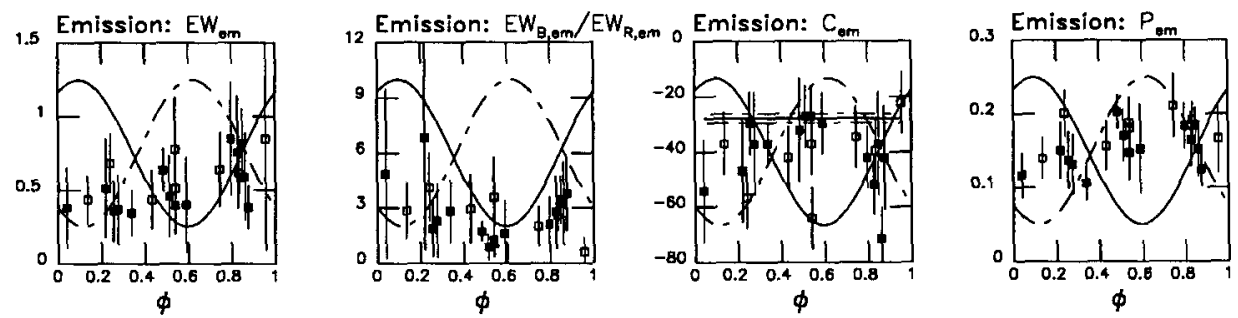

Fig. 2. The parameters $E W_{e m}, E W_{B, e m} / E W_{R, e m}, C_{e m}$ and $P_{e m}$. The notations are the same as in Fig 1.

tion of the mean brightness during the nine year cycle (Jetsu et al. , 1990a). The absence of detectable rotational modulation of $\mathrm{H} \alpha$ and of any correlation with the simultaneous light curve may thus be caused by the stronger axisymmetric component of spot distribution (i.e. spot activity is present on practically every observed longitude). Hence a detectable correlation with the light curve could only be expected when the axisymmetric part is weak compared non-axisymmetric part.

\section{References}

Bopp, B.W., Stencel, R.E.: 1981, Astrophys. J. Letters 247, 131

Bopp, B.W., Goodrich, B.D., Africano, J.L., Noah, P.V., Meredith, R.J., Palmer, L.H., Quigley, R.J.: 1984, Astrophys. J. 285, 202

Herbig, G. H.: 1958, Astrophys. J. 128, 295

Huenemoerder, D. P.: 1986, Astron. J. 92, 673

Jetsu, L., Huovelin, J., Tuominen, I., Vilhu, O., Bopp, B.W., Piirola, P.: 1990a, Astron. Astrophys. 236, 423

Jetsu, L., Huovelin, J., Tuominen, I., Bopp, B.W., Efimov, Yu.S., Linnaluoto, S., Nations, H. L., Piirola, P., Shakhovskoy, N.M., Virtanen, H.: 1990b, Astron. Astrophys. Suppl. 85, 813

Jetsu, L., Huovelin, J., Savanov, I., Tuominen, I.: 1990c, Astron. Astrophys., submitted Neff, J.E., Vilhu, O., Walter, F.M.: 1988, in A decade of UV Astronomy with IUE, ESA $S P-281,1 .$, p. 291

Walter, F.M., Basri, G.S.: 1982, Astrophys. J. 260, 735 